\title{
Noise-Removal Markers to Improve PCA-Based Face Recognition
}

\author{
Santiago-Omar Caballero-Morales \\ Technological University of the Mixteca \\ Road to Acatlima, Km. 2.5, Huajuapan de Leon, Oaxaca, Mexico, 69000 \\ scaballero@mixteco.utm.mx
}

\begin{abstract}
In this paper an approach based on insertion of "markers" is proposed to increase the performance of face recognition based on principal component analysis (PCA). The markers represent zero-valued pixels which are expected to remove information likely to affect classification (noisy pixels). The patterns of the markers was optimized with a genetic algorithm (GA) in contrast to other noise generation techniques. Experiments performed with a well known face database showed that the technique was able to achieve significant improvements on PCA particularly when data for training was small in comparison with the size of testing sets. This was also observed when the number of eigenfaces used for classification was small.
\end{abstract}

Keywords: face recognition, principal component analysis, genetic algorithms.

\section{Introduction}

Face recognition is one of the main problems studied in the field of pattern recognition. Achieving solutions for this problem has led to improvements in the development of technology for Human-Robot interaction, surveillance, automation, and interactive entertainment.

Among the most commonly used techniques for face recognition the following can be mentioned: Non-negative Matrix Factorization 214, Support Vector Machines (SVMs) and Principal Component Analysis (PCA) 35911. Performance under different conditions and test databases has been reported to be within the range of $95 \%$, 5 to $98 \%$ [3] for these techniques. Others as Artificial Neural Networks [6] and Hidden Markov Models (HMMs) [7] have been reported to achieve similar performance.

The work presented in this paper is aimed to provide a technique to improve the performance of PCA as a well known (and used) technique for face recognition. The proposed technique consists in the insertion of a set of "markers" in the form of zero-valued pixels to images to increase recognition. The type of distribution of markers required to achieve this goal without generating noise which would affect recognition is also studied by the present work. 
The paper is organized as follows. The details of the proposed technique within the context of PCA are presented in Section 2. Then in Section 3 the experiments performed with this technique on a well known database are presented and the obtained results are discussed. Finally in Section 4 the conclusions and future work are presented.

\section{Markers}

In the literature some works have presented the insertion of elements (e.g., noise) to improve the performance of learning techniques. In [13] the addition of Gaussian noise was proposed to improve the performance of Artificial Neural Networks (ANNs) for problems with small and unbalanced data sets. It was found that noise addition was able to reduce the risk of overfitting by adding more differences to the existing input patterns [1213].

In this work a different conception is considered and it is assumed that a pattern (in this case, an image) may already have elements (pixels) which may affect classification and should be removed. A "mark" which represents the removal of a "noisy" element (pixel) in an image by replacing it with a zero-valued pixel is proposed to improve the performance of a classification technique (in this case, PCA). This is different from considering additive or subtractive noise. Also, the distribution (positions) of these markers is considered to be specific for a particular input pattern, thus no assumption of Gaussian distribution is considered.

In practice, changing the value of an image's pixel to zero would be equivalent to add a mark to a person's face such as a mole. Such mark, in addition to other facial features, may support classification decisions. In Figure 1 the colored parametric-surfaces of an image from the faces94 database 910 are presented. In Figure 1(a) the original image is presented while in Figure 1(b) the original image with a group of zero-valued pixels in the left side is presented. The zerovalued pixels are clearly differentiable from most of the other pixels in the image. Hence the zero level is considered to be reliable to effectively "mark" an image.

\subsection{Integration of Markers}

The process for PCA training and testing in this work is the standard scheme presented in 3811 . In Figure 2 the overall process for the integration of the markers and evaluation of performance is presented. The markers are applied to each training image before the PCA process (leading to the markers being integrated into the estimation of the eigenfaces). These markers are also applied to each testing image before classification. Applying the markers is equivalent to the process of multiplying the vector representing the $i$ th face $\Gamma_{i}$ by a vector $X$ of the same size with ones (1's) and zeros (0's). In this way, the entries with " 1 " in $X$ preserve the associated pixel values in $\Gamma_{i}$, and the entries with " 0 " replace the associated pixel values in $\Gamma_{i}$. For illustration purposes the markers are presented as a matrix in Figure 2 where the black cells represent zeros (0's) and the white cells represent ones (1's). 


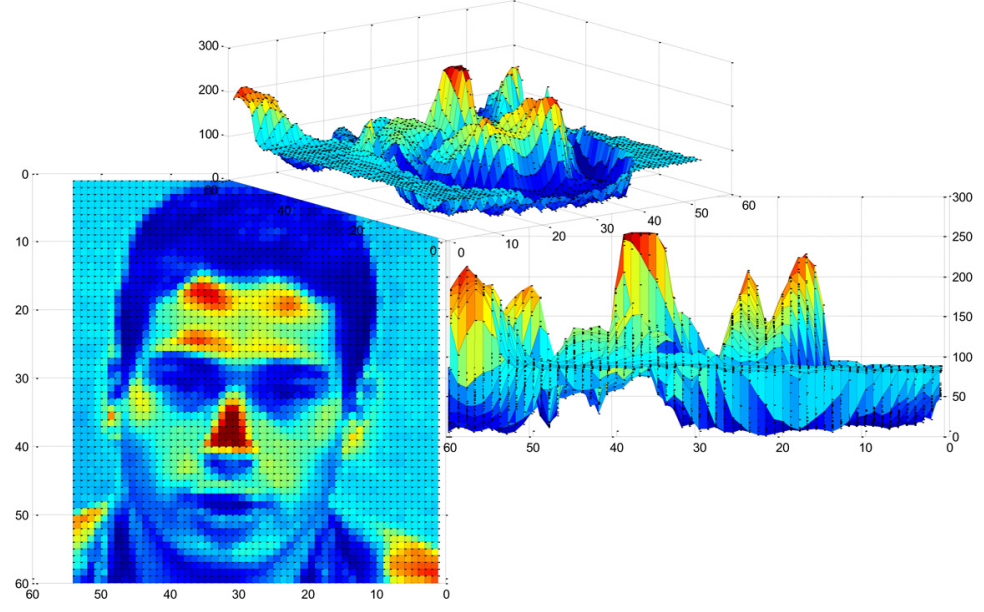

(a) Original

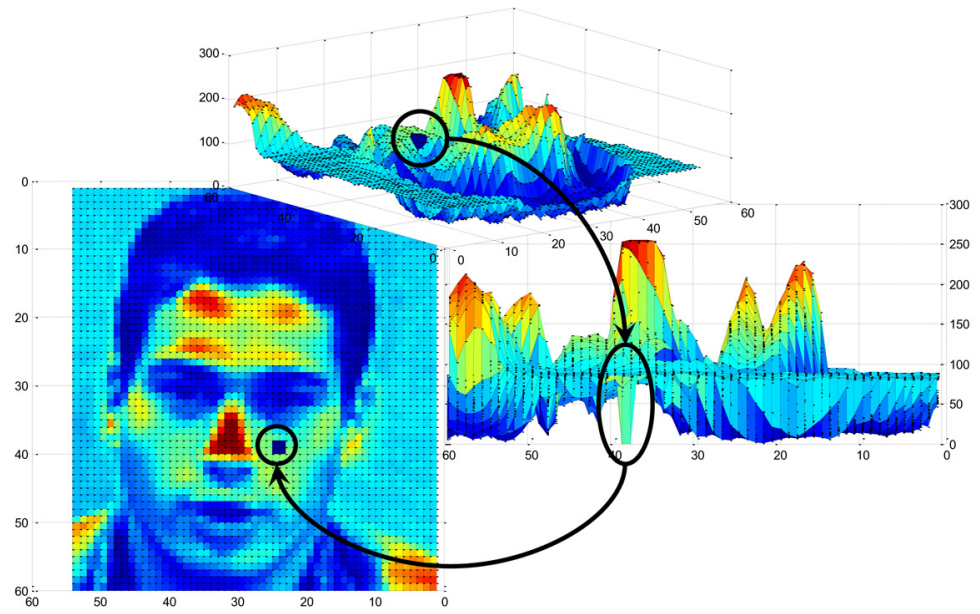

(b) Modified

Fig. 1. Effect of Replacing Pixels with Zero-Valued Markers 


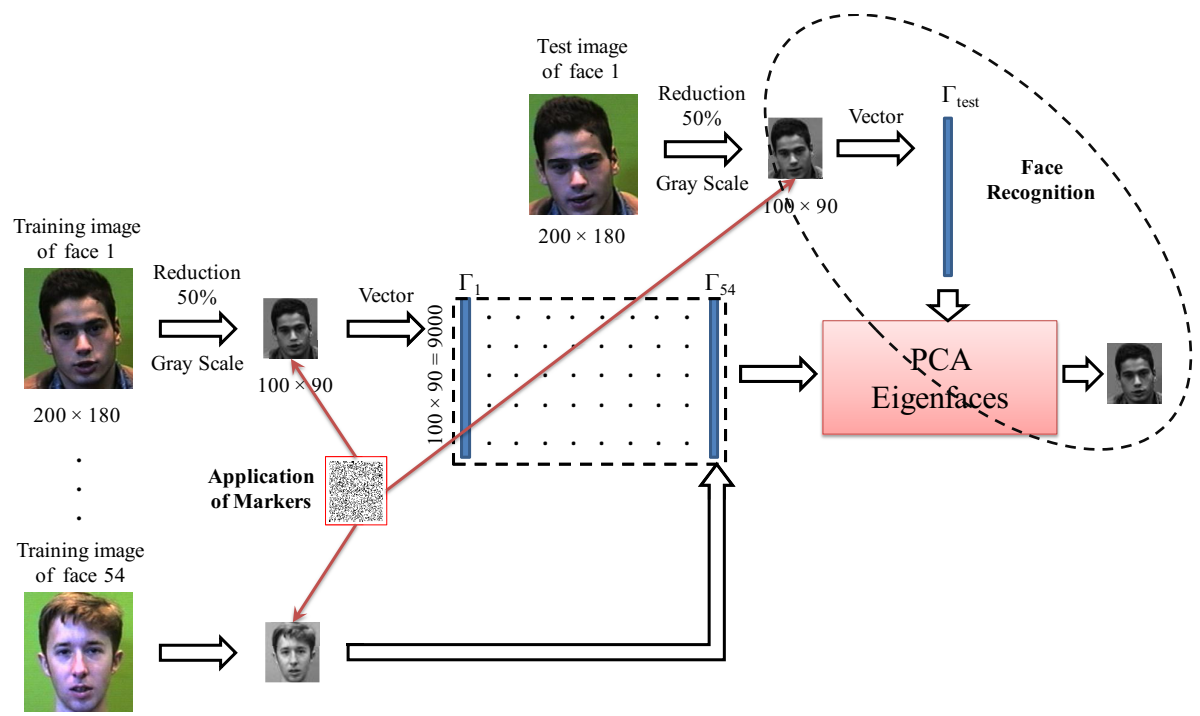

Fig. 2. Integration of Markers into the PCA Training and Testing Procedures

\section{$2.2 \quad$ Estimation of Markers}

The marker's distribution was estimated by means of a micro genetic algorithm $(\mu G A)$ [1] where the chromosome consists of an $X$ vector with " 1 " and " 0 " entries. The fitness value was measured as the PCA recognition performance obtained over a subset of test images considering the application of the $X$ vector on these images and the training images. The use of a $\mu G A$ was considered given the size of the chromosome ( $n$ columns $\times m$ rows) and also because it can converge quickly within a few iterations with a very small initial population, providing estimates as good as a conventional GA. The structure and parameters of the $\mu G A$ are presented in Figure 3 .

\section{$3 \quad$ Experiments and Results}

\subsection{Face Database}

For this work the faces94 database was used [910]. This database consists of images from 153 individuals (20 females, 133 males) with a resolution of 180 by 200 pixels (portrait format). A total of 20 images from each individual are available in the database. These images have some minor variations between them as small changes in head turn, tilt and slant, position of face in image, and expressions.

For the experiments 54 individuals were randomly selected, and the set of images from each individual was separated into the following subsets:

- Images 1 to 4: for training scheme (TS) 1 (training with 4 images).

- Images 1 to 8: for training scheme (TS) 2 (training with 8 images). 


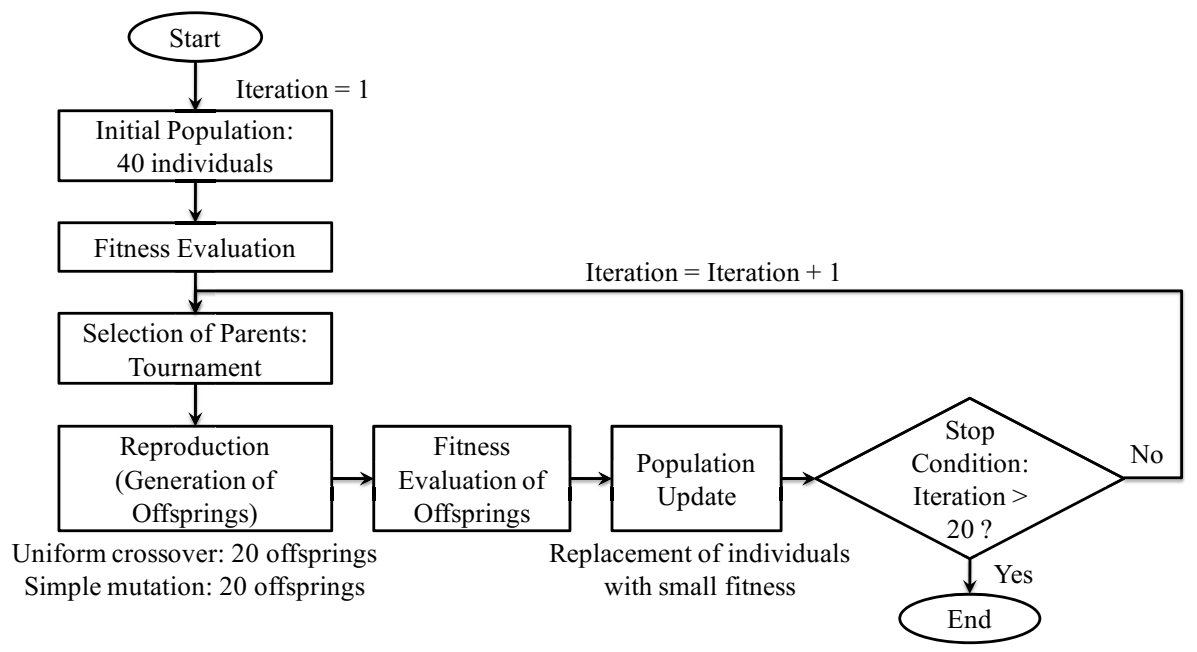

Fig. 3. Structure and Parameters of the Estimation Algorithm

- Images 9 to 12: for fitness evaluation of the GA.

- Images 13 to 20: for testing (recognition performance).

As presented two training schemes were considered. This was performed in order to evaluate the performance of the proposed technique with small and medium training data. Note that under the training scheme TS 2, both training and testing sets are equally sized. In contrast, under the training scheme TS 1 the testing set is twice the size of the training set.

Also the effect of the proposed technique on PCA performance was explored when the number of eigenfaces is varied. For this, the following numbers of eigenfaces were considered: $1,4,8,12$ and 16 .

The results considering these conditions are presented and discussed in the following sections.

\subsection{Convergence of the $\mu G A$}

In Figure 4 the mean convergence plots of the $\mu G A$ for the 54 individuals using 4 and 8 training images and different number of eigenfaces are presented.

Under both training schemes improvement in recognition is obtained with the use of the markers (PCA-Markers) when compared with the performance of the standard PCA (PCA-Original).

\subsection{Distribution of Markers}

In Table 1 the patterns (distribution) of the markers estimated with the $\mu G A$ are presented for each experimental condition. Considering that each image consists of $100 \times 90$ pixels $=9000$ pixels (after reduction), some markers consists of approximately $50 \%$ of the total number of pixels of an image. 


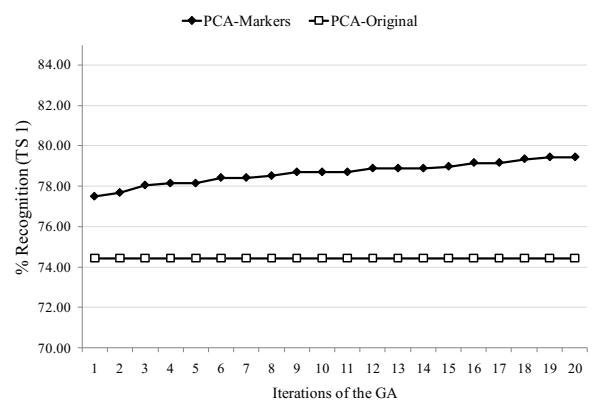

(a) TS 1

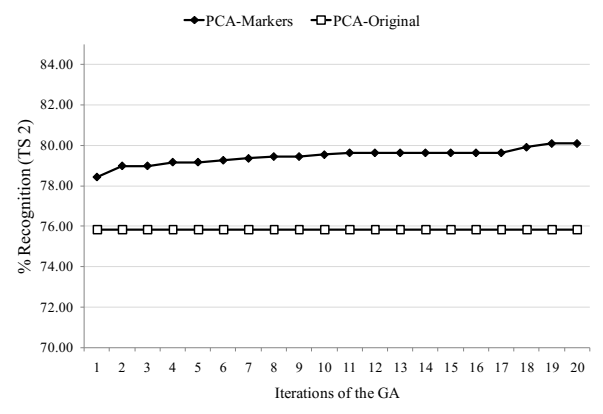

(b) TS 2

Fig. 4. Mean Convergence Plots of the $\mu G A$ for Different Training Schemes and Number of Eigenfaces

Table 1. Distribution of Markers Estimated with the $\mu G A$

\begin{tabular}{|c|c|c|c|c|c|}
\hline Train & \multicolumn{5}{|c|}{ Number of Eigenfaces } \\
\hline TS 1 & 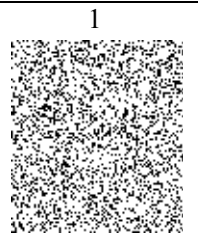 & 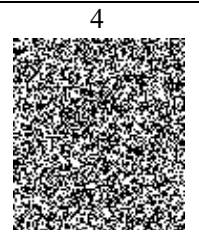 & 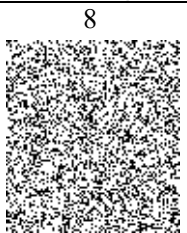 & 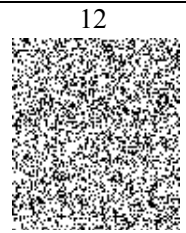 & 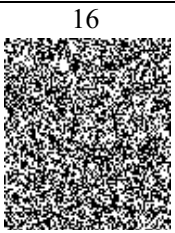 \\
\hline & 2271 markers & 4489 markers & 2845 markers & 2908 markers & 4568 markers \\
\hline TS 2 & 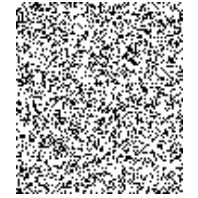 & 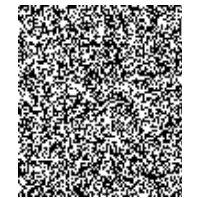 & 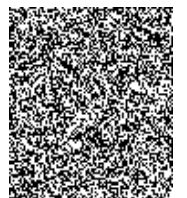 & 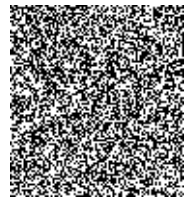 & 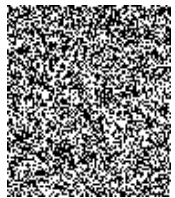 \\
\hline & 2819 markers & 4430 markers & 4491 markers & 4540 markers & $39 \mathrm{~m}$ \\
\hline
\end{tabular}

It is important to mention that no clear structures are presented in these patterns, and thus these may look like randomly generated patterns. To address this situation a comparison of the markers' distributions with randomly generated binary matrices was performed. Two random binary matrices, RndMat 1 and RndMat 2, were created for this purpose using a continuous uniform distribution.

If a marker's pattern $\mathrm{Z}$ is similar to a randomly generated matrix RndMat 1 (or RndMat 2), it is expected that the distance between $\mathrm{Z}$ and RndMat 1 (or RndMat 2) be similar to the distance between two randomly generated matrices (RndMat 1 and RndMat 2). In contrast, if $\mathrm{Z}$ is not similar to a randomly generated matrix, then the distance between $\mathrm{Z}$ and RndMat 1 (or RndMat 2) must be higher than the distance between two randomly generated matrices (RndMat 1 and RndMat 2). In Table 2 the normalized Earth Mover's Distances (EMD) between the random matrices and the markers distributions from Table 1 are presented. 
Table 2. Distances between Randomly Generated Patterns and the Markers

\begin{tabular}{|c|c|cccccc|}
\hline Reference & \multicolumn{7}{|c|}{ Comparison Patterns } \\
\cline { 2 - 8 } Pattern & Train & \multicolumn{7}{|c|}{ Number of Eigenfaces } \\
\hline & & 1 & 4 & 8 & 12 & 16 & RndMat 2 \\
RndMat 1 & TS 1 & 3.14 & 1.00 & 2.49 & 2.25 & 1.00 & 1.00 \\
& TS 2 & 2.38 & 0.99 & 1.02 & 0.96 & 0.96 & 1.00 \\
\hline
\end{tabular}

Taking as reference the EMD distance between the random matrices RndMat 1 and RndMat 2, it is observed that the markers estimated with 4 training images (TS 1) and 4 and 16 eigenfaces have very similar distance to the random matrix RndMat 1. For the same training scheme the markers estimated with 1,8 , and 12 eigenfaces have a higher distance to the random matrix RndMat 1. From Table 1 it is observed that these markers have the lowest number of elements (2271, 2845, and 2908 respectively).

For the case with 8 training images (TS 2) the highest distance is obtained with one eigenface (2819 elements) while the similar distance to the random matrix RndMat 1 is obtained with the markers estimated for 4, 8, 12 and 16 eigenfaces (4430, 4491, 4540, and 4489 elements respectively). As the number of markers increases their distribution seems to resemble a random binary matrix. The highest distance from a random pattern (3.14) is obtained with the distribution with the lowest number of markers (2271). Thus, for initial evaluation of performance the pattern with the minimum number of markers was used.

\subsection{PCA Performance}

The performance of PCA with the selected pattern of markers (PCA-Markers) over the test set of 8 images is presented in Figures $5(\mathrm{a})$ and $5(\mathrm{~b})$ for the training schemes TS 1 and TS 2. The numerical data associated to these figures is presented in Table 3 .

While the mean gains observed in Figures 5(a) and 5(b) may look significant for small number of eigenfaces, when performing a technique of statistical analysis [4] it was found that some of these were not statistically significant. As presented in Table 3 for the case where PCA was trained with 4 images (TS 1), the improvement was statistically significant at the 0.10 level when one eigenface was used. On the other hand the improvement was marginally significant at the 0.15 level when 8 and 12 eigenfaces were used. When PCA was trained with 8 images (TS 2), the improvement was statistically significant at the 0.05 , 0.10 , and 0.15 levels when 4,8 and 12 eigenfaces were used. Considering that the markers were those estimated with the PCA trained with 4 images (TS 1), statistically significant improvements were obtained in a PCA system trained with more data (TS 2). 


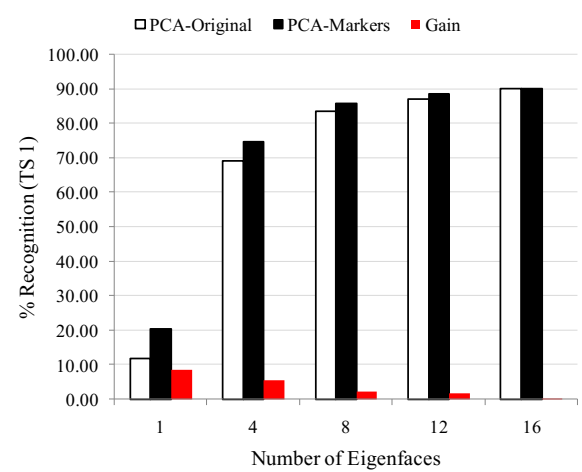

(a) TS 1

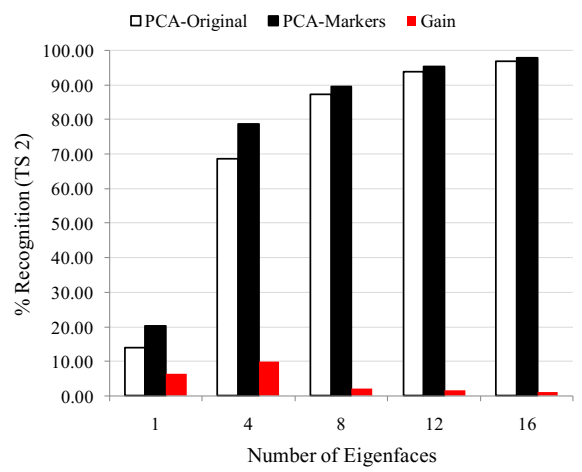

(b) TS 2

Fig. 5. Mean Recognition Performance on the Test Set (Histogram)

Table 3. Mean Recognition Performance on the Test Set (Numerical Data)

\begin{tabular}{|cccccl|}
\hline Train & Eigenfaces & PCA-Original & PCA-Markers & p-value & Conclusion \\
\hline & 1 & 11.81 & 20.37 & 0.0990 & $<0.10$ \\
\multirow{4}{*}{ TS 1} & 4 & 68.98 & 74.54 & 0.2033 & Not Significant \\
& 8 & 83.33 & 85.65 & 0.1447 & $<0.15$ \\
& 12 & 86.81 & 88.43 & 0.1218 & $<0.15$ \\
& 16 & 89.81 & 90.05 & 0.8201 & Not Significant \\
\hline \multirow{4}{*}{ TS 2} & 1 & 13.89 & 20.14 & 0.2632 & Not Significant \\
& 4 & 68.75 & 78.70 & 0.0091 & $<0.05$ \\
& 8 & 87.50 & 89.58 & 0.0774 & $<0.10$ \\
& 12 & 93.98 & 95.37 & 0.1035 & $<0.15$ \\
& 16 & 96.99 & 97.92 & 0.2033 & Not Significant \\
\hline
\end{tabular}

\section{Future Work}

In this paper an approach based on removal of (possibly) noisy pixels by the insertion of zero-valued pixels (markers) was presented. The approach was able to obtain statistically significant improvements on PCA for face recognition under different conditions of size of training data and number of eigenfaces. It is of particular attention that the test set was of equal size (and also larger) than the size of the training set.

Although these results may look encouraging it is very important to mention the exploratory nature of this work. Thus more extensive experiments must be performed to address the significance of these results. Among the ongoing and future work the following points are considered:

- to evaluate the performance of the technique with the complete faces94 database and analyze the effect of all patterns of markers on the recognition process; 
- to integrate the technique with other recognition techniques as NMF, ANNs, and HMMs;

- to develop an algorithm to define a threshold for the number of markers.

\section{References}

1. Bakare, G.A., Venayagagamoorthy, G.K., Aliyu, U.O.: Reactive Power and Voltage Control of the Nigerian Grid System Using Micro-Genetic Algorithm. In: Proc. of the Power Engineering Society General Meeting, vol. 2, pp. 1916-1922 (2005)

2. Chen, W.S., Pan, B., Fang, B., Li, M., Tang, J.: Incremental Nonnegative Matrix Factorization for Face Recognition. In: Mathematical Problems in Engineering, pp. 1-17 (2008)

3. Faruqe, M.O., Al Mehedi Hasan, M.: Face recognition using PCA and SVM. In: Proc. of 3rd International Conference on Anti-counterfeiting, Security, and Identification in Communication (ASID 2009), pp. 97-101 (2009)

4. Gillick, L., Cox, S.J.: Some statistical issues in the comparison of speech recognition algorithms. In: Proc. IEEE Conf. on Acoustics, Speech and Signal Processing (ICASSP 1989), vol. 1, pp. 532-535 (1989)

5. Heisele, B., Ho, P., Poggio, T.: Face recognition with support vector machines: Global versus component-based approach. In: Proc. of 8th IEEE International Conference on Computer Vision (ICCV 2001), vol. 2, pp. 688-694 (2001)

6. Islam, M.R., Toufiq, R., Sobhan, M.A.: Appearance and shape based face recognition using Backpropagation learning neural network algorithm with different lighting variations. Science Journal of Circuits, Systems and Signal Processing 2(4), 93-99 (2013)

7. Miar-Naimi, H., Davari, P.: A New Fast and Efficient HMM-Based Face Recognition System Using a 7-State HMM Along With SVD Coefficients. Iranian Journal of Electrical \& Electronic Engineering 4(1), 46-57 (2008)

8. Moon, H., Phillips, P.J.: Computational and performance aspects of PCA-based face-recognition algorithms. Perception 30, 303-321 (2001)

9. Poon, B., Ashraful Amin, M., Yan, H.: Performance evaluation and comparison of PCA Based human face recognition methods for distorted images. International Journal of Machine Learning and Cybernetics 2, 245-259 (2011)

10. Spacek, L.: Collection of facial images: Faces94. In: Computer Vision Science and Research Projects, University of Essex, United Kingdom, http://cswww.essex.ac.uk/mv/allfaces/faces94.html

11. Turk, M., Pentland, A.: Eigenfaces for Recognition. Journal of Cognitive Neuroscience 3(1), 71-86 (1991)

12. Viegas da Silva, I.B., Adeodato, J.L.: An Approach for Learning from Small and Unbalanced Data Sets using Gaussian Noise during Artificial Neural Networks Training. In: Proc. of International Conference on Data Mining (DMIN 2010), pp. 23-30 (2010)

13. Viegas da Silva, I.B., Adeodato, J.L.: PCA and Gaussian Noise in MLP Neural Network Training Improve Generalization in Problems with Small and Unbalanced Data Sets. In: Proc. of International Joint Conference on Neural Networks (IJCNN 2011), pp. 2664-2669 (2011)

14. Xue, Y., Tong, C.S., Zhang, W.: Survey of Distance Measures for NMF-Based Face Recognition. In: Wang, Y., Cheung, Y.-M., Liu, H. (eds.) CIS 2006. LNCS (LNAI), vol. 4456, pp. 1039-1049. Springer, Heidelberg (2007) 